\title{
Erratum to: Associations between energy intake, daily food intake and energy density of foods and BMI $z$-score in 2-9 year old European children
}

\author{
A. Hebestreit - C. Börnhorst - G. Barba - A. Siani - I. Huybrechts • \\ G. Tognon - G. Eiben - L. A. Moreno - J. M. Fernández Alvira • \\ H. M. Loit $\cdot$ E. Kovacs $\cdot$ M. Tornaritis $\cdot$ V. Krogh
}

Published online: 10 May 2014

(c) Springer-Verlag Berlin Heidelberg 2014

\section{Erratum to: Eur J Nutr (2014) 53:673-681 \\ DOI 10.1007/s00394-013-0575-x}

In the original publication of this article, there was an error in the last line of Table 3: "Interaction between daily energy and food intake" should be written instead of "Energy density of foods (kcal/g)".

This does not affect description of analysis, result or outcome.

The corrected version of Table 3 is shown below.

The online version of the original article can be found under doi:10.1007/s00394-013-0575-x.

\section{A. Hebestreit $(\square) \cdot$ C. Börnhorst}

Department of Epidemiological Methods and Etiologic

Research, Leibniz Institute for Prevention Research and

Epidemiology, BIPS GmbH, Achterstrasse 30,

28359 Bremen, Germany

e-mail: hebestr@bips.uni-bremen.de

G. Barba $\cdot$ A. Siani

Institute of Food Sciences, National Research Council,

Avellino, Italy

I. Huybrechts

Department of Public Health, Ghent University, Ghent, Belgium

I. Huybrechts

Dietary Exposure Assessment Groups, International Agency

for Research on Cancer, Lyon, France

G. Tognon · G. Eiben

Department of Public Health and Community Medicine,

University of Gothenburg, Göteborg, Sweden
L. A. Moreno · J. M. Fernández Alvira

GENUD (Growth, Exercise, Nutrition and Development)

Research Group, School of Health Sciences,

University of Zaragoza, Zaragoza, Spain

H. M. Loit

Department of Chronic Diseases, National Institute

for Health Development, Tallinn, Estonia

\section{E. Kovacs}

Department of Pediatrics, University of Pécs, Pecs, Hungary

M. Tornaritis

Research and Education Institute of Child Health,

Strovolos, Cyprus

V. Krogh

Department of Preventive and Predictive Medicine, Fondazione IRCCS Istituto Nazionale dei Tumori, Milan, Italy 
Table 3 Associations between energy intake, daily food intake and energy density of foods with BMI $z$-score adjusted for age, sex and ISCED level and including study center as random effect

\begin{tabular}{|c|c|c|c|c|c|c|c|}
\hline \multicolumn{4}{|l|}{ Full sample $(N=9,782)$} & \multicolumn{4}{|c|}{ Plausible energy reports $(N=8,544)$} \\
\hline Parameter & Estimate & $\begin{array}{l}\text { Standard } \\
\text { error }\end{array}$ & $P$ value & Parameter & Estimate & $\begin{array}{l}\text { Standard } \\
\text { error }\end{array}$ & $P$ value \\
\hline Model $1 a^{\mathrm{a}}$ & & & & Model $1 b^{\mathrm{b}}$ & & & \\
\hline Intercept & -0.560 & 0.137 & 0.004 & Intercept & -0.878 & 0.132 & 0.0003 \\
\hline $\begin{array}{l}\text { Daily energy intake } \\
\quad(1 \text { unit } \sim 100 \mathrm{kcal})\end{array}$ & -0.002 & 0.003 & 0.427 & $\begin{array}{l}\text { Daily energy intake } \\
\quad(1 \text { unit } \sim 100 \mathrm{kcal})\end{array}$ & 0.032 & 0.004 & $<0.0001$ \\
\hline Model $2 a^{\mathrm{a}}$ & & & & Model $2 b^{\mathrm{b}}$ & & & \\
\hline Intercept & -0.623 & 0.138 & 0.003 & Intercept & -0.794 & 0.147 & 0.001 \\
\hline $\begin{array}{l}\text { Daily food intake } \\
\quad(1 \text { unit } \sim 100 \mathrm{~g})\end{array}$ & 0.0037 & 0.0033 & 0.297 & $\begin{array}{l}\text { Daily food intake } \\
\quad(1 \text { unit } \sim 100 \mathrm{~g})\end{array}$ & 0.027 & 0.004 & $<0.0001$ \\
\hline Model $3 a$ & & & & Model $3 b^{b}$ & & & \\
\hline Intercept & -0.520 & 0.143 & 0.008 & Intercept & -0.555 & 0.139 & 0.005 \\
\hline Energy density of foods (kcal/g) & -0.056 & 0.037 & 0.131 & $\begin{array}{l}\text { Energy density of } \\
\text { foods }(\mathrm{kcal} / \mathrm{g})\end{array}$ & 0.042 & 0.040 & 0.302 \\
\hline Model $4 a^{\mathrm{a}}$ & & & & Model $4 b^{\mathrm{b}}$ & & & \\
\hline Intercept & -0.450 & 0.167 & 0.031 & Intercept & -0.956 & 0.195 & 0.002 \\
\hline $\begin{array}{l}\text { Daily energy intake } \\
\quad(1 \text { unit } \sim 100 \mathrm{kcal})\end{array}$ & -0.0191 & 0.007 & 0.007 & $\begin{array}{l}\text { Daily energy intake } \\
\quad(1 \text { unit } \sim 100 \mathrm{kcal})\end{array}$ & 0.030 & 0.010 & 0.002 \\
\hline $\begin{array}{l}\text { Daily food intake } \\
\quad(1 \text { unit } \sim 100 \mathrm{~g})\end{array}$ & -0.002 & 0.009 & 0.839 & $\begin{array}{l}\text { Daily food intake } \\
\quad(1 \text { unit } \sim 100 \mathrm{~g})\end{array}$ & 0.012 & 0.013 & 0.321 \\
\hline $\begin{array}{l}\text { Interaction between daily energy } \\
\text { and food intake }\end{array}$ & 0.001 & 0.001 & 0.076 & $\begin{array}{l}\text { Interaction between daily } \\
\text { energy and food intake }\end{array}$ & -0.0002 & 0.001 & 0.740 \\
\hline
\end{tabular}

Models 1-3: association between exposure variables and BMI $z$-score were investigated in separate models

Models 4: association between exposure variables and BMI $z$-score were investigated in a combined model including an additional interaction term

${ }^{a}$ Effects of the dietary variables in the full sample

${ }^{b}$ Effects of the dietary variables in the sub-sample of plausible energy reports 